\title{
Hiding the Higgs at the LHC
}

\author{
Omri Bahat-Treidel $*$ Yuval Grossman $\dagger$ and Yoram Rozen $\ddagger$ \\ Department of Physics, Technion-Israel Institute of \\ Technology, Technion City, Haifa 32000, Israel
}

\begin{abstract}
We study a simple extension of the standard model where scalar singlets that mix with the Higgs doublet are added. This modification to the standard model could have a significant impact on Higgs searches at the LHC. The Higgs doublet is not a mass eigenstate and therefore the expected nice peak of the standard model Higgs disappears. We analyze this scenario finding the required properties of the singlets in order to make the Higgs "invisible" at the LHC. In some part of the parameter space even one singlet could make the discovery of the SM Higgs problematic. In other parts, the Higgs can be discovered even in the presence of many singlets.
\end{abstract}

*Electronic address: bahat@techunix.technion.ac.il

$\dagger$ Electronic address: yuvalg@physics.technion.ac.il

${ }^{\ddagger}$ Electronic address: rozen@techunix.technion.ac.il 


\section{INTRODUCTION}

The Higgs particle of the Standard Model (SM) is expected to be discovered at the LHC. In extensions of the standard model, however, the situation could be different. Modifications to the scalar sector alter the experimental signatures of the Higgs boson in a model dependent way. Therefore, there is no guarantee that a very general Higgs boson can be found at the LHC.

The available experimental data provide constraints on the Higgs mass, $m_{H}$ (for a review see [1]). The strongest lower bound comes from direct searches at LEP2, $m_{H}>114.4 \mathrm{GeV}$ at 95\% CL [2]. An upper bound is derived from electroweak precision measurements and reads $m_{H}<219 \mathrm{GeV}$ at $95 \% \mathrm{CL}[3]$. Since the sensitivity of electroweak precision measurements to $m_{H}$ is logarithmic, we cannot exclude at a very high confidence level the case where $m_{H}$ is just a factor of a few above this limit.

One of the main goals of the LHC is to discover the Higgs boson. Both the ATLAS and CMS collaborations will search for the Higgs boson in the mass range of $10^{2}-10^{3} \mathrm{GeV}$. The Higgs is expected to be discovered through different channels depending on its mass. In the low mass regime, the most promising channel would be $H \rightarrow \gamma \gamma$ [4]. For $m_{H} \gtrsim 150 \mathrm{GeV}$, the preferred decay is $H \rightarrow V V^{(*)}$ (with $V=Z, W$ ) with different substantial decays of the vector bosons. These searches are expected to provide at least a $5 \sigma$ signal for the Higgs after few years of operation of the LHC.

There are also several theoretical constraints on $m_{H}$ (see, for example, [5]). For example, the unitarity bound reads $m_{H} \lesssim 700 \mathrm{GeV}$. One can also consider the possibility that the Higgs does not exist. This possibility gives rise to a constraint on new physics scale $\Lambda \lesssim 1 \mathrm{TeV}$. Thus, we expect that the LHC will find either the Higgs boson or some kind of new physics.

What if nothing is found at the LHC, that is, neither the Higgs boson nor new physics? Such a scenario seems to imply that $(i)$ the Higgs boson does not exist; ( $i i)$ there is new physics that is responsible for electroweak-symmetry breaking (EWSB); and (iii) the experimental signals of this new physics are such that it cannot be discovered at the LHC. There is, however, another possibility: The Higgs exists and it is responsible for EWSB but there is new physics that "hides" the Higgs signals. Furthermore, this new physics does not show up in any other channel and therefore cannot be discovered at the LHC.

Here we study such a scenario which hides the Higgs and does not show any signal of new physics. We extend the scalar sector of the SM by introducing additional SM singlets which mix with the Higgs doublet of the SM. The resulting spectrum consists of many scalars. Each of these scalars is mainly a singlet with a small component of the SM doublet. Thus, the production rate for any of these mass eigenstates is much smaller than that of a SM Higgs with the same mass. In the limit of many singlets each mass eigenstate produces a very small signal that cannot be separated from the background. In that case the Higgs is practically hidden. 
While our model is phenomenologically interesting, and demonstrate how surprises may occur at the LHC due to simple new physics, it has no clear theoretical motivation. In particular, while the model is not necessarily fine-tuned, there is no reason why the new singlets have weak scale masses and vevs. Our motivation is to study the phenomenology of the model. Thus, at this stage, we do not try to find a motivated fundamental framework that accommodates our model as its low energy limit.

Several papers that study similar ideas has been published. A model very similar to our was study by Espinosa and Gunion [6]. Like us, they study the effect of adding many singlets to the SM. However, they focus on a $\sqrt{s}=500 \mathrm{Gev}$ linear collider. They found that such a multi singlet Higgs sector can be detected at such a machine.

Singlet extensions of the SM where also discussed in other papers with emphasis more on the effect of invisible decays of the Higgs. Ref. [7] discusses an $\mathcal{O}(\mathrm{N})$ model without SSB of the internal symmetry, and therefore there is no mixing with the Higgs. In [8] a model with complex gauge singlet was studied. The focus in that work had been put on the invisible decay of the Higgs into the singlets goldstone modes. Refs. [9, 10] have studied a SM replica called phantom sector. The phantom doublet acts as SM singlet which might change the experimental signature. Two scenarios were examined. The first, there is no mixing with the Higgs and the only effect is the invisible decay (see also [11]). The second, such mixing does exist and the affect is reduced production rate.

Ref. [12] explains an excess above LEP2 background with large number of higher dimensional singlet scalar fields, which mix with the SM Higgs. This model implies that none of the search channels would work, while our analysis present a different picture. In Ref. [13, 14] it has been showed that the Higgs might have been missed at LEP2. This possibility rises from the NMSSM, where the Higgs decays into two light $\mathcal{C P}$ odd Higgs bosons.

Ref. [15] study a model similar to ours. Yet, while we concentrate on the case of many singlets with large mixing with the SM Higgs, [15] studies mainly the case of one singlet with a small mixing angle. In Ref. [16, 17] effective new operators were introduced. These new operators can also hide the Higgs from being discovered without showing any signal of new physics, but the mechanism is different from ours.

Supersymmetric versions of our model study in Ref. [18, 19, 20, 21] where signals of a supersymmetric model with one extra singlet were investigated. Another supersymmetric model with a splitted Higgs signal have been used to explain excesses of a Higgs signal in LEP2 [22]. Our model is not supersymmetric and the collider signatures are different than those discussed in these papers.

\section{THE MODEL}

In order to understand the main features of our scenario we start with a simple case where one singlet, $S(1,1)_{0}$, is added to the SM. For simplicity we further introduce a $Z_{2}$ symmetry 
such that $S$ is odd under it, while all other fields are even under this $Z_{2}$. Denoting the SM Higgs doublet by $H$, the most general renormalizable scalar potential is

$$
\mu_{H}^{2}|H|^{2}+\frac{\mu_{S}^{2}}{2} S^{2}+\lambda_{H}|H|^{4}+\frac{\lambda_{S}}{4} S^{4}+\frac{\eta}{2} S^{2}|H|^{2} .
$$

In the following we assume that

$$
\mu_{H} \sim \mu_{S}, \quad \lambda_{H} \sim \lambda_{S} \sim \eta
$$

While our assumptions, that all dimensionful parameters are at the same scale and all dimensionless couplings are of the same order, are simple and not necessarily fine-tuned, they are not based on a fundamental framework of new physics. We make them because they lead to interesting phenomenology.

We are interested in the vacuum structure of this potential. Since the Higgs vev is responsible for EWSB we demand $\langle H\rangle \neq 0$. As for the vev of $S$, the solution $\langle S\rangle=0$ is not interesting as there is no mixing between $S$ and $H$. Thus, we consider only solutions where $\langle H\rangle \neq 0$ and $\langle S\rangle \neq 0$. It is worth mentioning that in general there is a large part of the parameter space where both fields acquire a vev.

Next, we analyze the mass spectrum. We substitute

$$
\mathcal{R} e(H) \rightarrow \frac{h+v_{H}}{\sqrt{2}}, \quad S \rightarrow s+v_{S}
$$

where $h$ and $s$ are real scalar fields and $v_{H}$ and $v_{S}$ are the vacuum expectation values of $H$ and $S$ respectively. The mass-squared matrix in the $(h, s)$ basis is

$$
M^{2}=\left(\begin{array}{cc}
\mu_{H}^{2}+3 \lambda_{H} v_{H}^{2}+\frac{1}{2} \eta v_{S}^{2} & \eta v_{H} v_{S} \\
\eta v_{H} v_{S} & \mu_{S}^{2}+3 \lambda_{S} v_{S}^{2}+\frac{1}{2} \eta v_{H}^{2}
\end{array}\right)
$$

Diagonalizing $M^{2}$, we get two mass eigenstates, $\phi_{0}$ and $\phi_{1}$ with masses $m_{0}$ and $m_{1}$. We define $m_{0} \leq m_{1}$ and due to our assumptions we expect $m_{0} \sim m_{1}$. We further consider only cases where the two mass eigenstates are not close to be degenerate, that is, $m_{1}-m_{0} \gg \Gamma_{0}, \Gamma_{1}$. The two mass eigenstates are related to the weak eigenstates $h$ and $s$ by a $2 \times 2$ orthogonal rotation matrix $V$

$$
\left(\begin{array}{l}
h \\
s
\end{array}\right)=V\left(\begin{array}{l}
\phi_{0} \\
\phi_{1}
\end{array}\right), \quad V=\left(\begin{array}{cc}
\cos \theta & \sin \theta \\
-\sin \theta & \cos \theta
\end{array}\right) .
$$

Note that $\theta$ can assume any value between 0 and $\pi / 2$. In general $\theta$ can be very small, but due to our assumption, Eq. (2), we expect $\theta \sim O(1)$. The model discussed here contains five parameters. They can be chosen to be the five parameters in (1). Instead, we can chose them to be the two masses, $m_{0}$ and $m_{1}$, the two vevs, $v_{S}$ and $v_{H}$ and the mixing angle $\theta$.

We are now in position to study the phenomenology of the model. The couplings of the scalars to the SM fields can be obtained from that of the SM Higgs by projecting onto the 
doublet component. In particular, we are interested in the coupling of a scalar to a pair of SM fields, either fermions or vector bosons

$$
\frac{V_{h i}}{v_{H}}\left(m_{f} \phi_{i} \bar{f} f+m_{Z}^{2} \phi_{i} Z_{\mu} Z^{\mu}+2 m_{W}^{2} \phi_{i} W_{\mu}^{+} W^{\mu-}\right) \text {. }
$$

We see that the couplings are just the SM couplings projected by $V_{h i}$. The couplings between two scalars and two gauge bosons are given by the SM ones multiplied by $V_{h i} V_{h j}$

$$
\frac{V_{h i} V_{h j}}{2 v_{H}^{2}}\left(m_{Z}^{2} \phi_{i} \phi_{j} Z_{\mu} Z^{\mu}+2 m_{W}^{2} \phi_{i} \phi_{j} W_{\mu}^{+} W^{\mu-}\right) \text {. }
$$

Last we need the self interactions term, i.e., interaction that involve only scalars. The interesting part for our study is the couplings that can be responsible for decays of a heavy scalar into light scalars, $\phi_{1} \rightarrow 2 \phi_{0}$ and $\phi_{1} \rightarrow 3 \phi_{0}$. These couplings are given by

$$
\begin{aligned}
& \frac{1}{4}\left[\left(\lambda_{S}-\lambda_{H}-\left(\lambda_{S}+\lambda_{H}-\eta\right) \cos 2 \theta\right) \sin 2 \theta\right] \phi_{1} \phi_{0}^{3}+ \\
& {\left[v_{\phi} \cos \theta\left(\left(3 \lambda_{S}-\eta\right) \sin ^{2} \theta+\frac{\eta}{2} \cos ^{2} \theta\right)-v_{H} \sin \theta\left(\left(3 \lambda_{H}-\eta\right) \cos ^{2} \theta+\frac{\eta}{2} \sin ^{2} \theta\right)\right] \phi_{1} \phi_{0}^{2} .}
\end{aligned}
$$

In general there are no specific relations between the strength of the scalar couplings, Eq. (8)), and the couplings between scalars and gauge bosons, Eqs. (6) and (7). For example, the coupling of $\phi_{1} \phi_{0}^{2}$ can be similar, smaller or larger to that of $\phi_{1} W^{+} W^{-}$.

We can generalize the above model by introducing $N$ new singlets, $S_{\alpha}$, with $\alpha=1 . . N$. Again, we analyze the most interesting case where all the scalar fields acquire vevs. The algebra is more cumbersome, but we end up with a result similar to the case of one extra singlet. There are $N+1$ mass eigenstates $\phi_{i}(i=0 . . N)$. We expand around the vacuum in a similar way as Eq. (3) . In terms of the weak eigenstates, $\phi^{W} \equiv\left(h, s_{\alpha}\right)$, the mass eigenstates $\phi$ are given by $\phi=V \phi^{W}$, such that $V$ is an $(N+1) \times(N+1)$ orthogonal matrix. The couplings to the SM fields are then given as in the one singlet case by Eqs. (6) and (17). The analog of Eq. (8) is more complicated. It can be obtained in a straightforward way and we do not write it explicitly here. We only mention that also in the more general case considered here the couplings between the scalars can be smaller, similar, or larger with respect to other couplings which involves gauge bosons.

\section{PHENOMENOLOGY OF THE MODEL}

Next we study the phenomenology of the $N$ singlets model. We first look at the effect of this model on electroweak precision measurements (see also [8]) and then move to discuss the collider signatures.

The SM Higgs contribution to electroweak precision measurements comes through the $S$ and $T$ parameters [23]. That is, the gauge boson self energies are the only numerically relevant diagrams with the Higgs. Of course varying $m_{H}$ affects all observables, but in a 
way consistent with changing just $S$ and $T$. Thus, in order to see the effects of our model, all we need to do is to replace the SM Higgs contributions to $S$ and $T$ with the sum of all contributions weighted by the mixing angles. Consider a one-loop diagram with the $i$ th mass eigenstate. Its contribution to $S$ and $T$ is equal to that of the corresponding SM diagram multiplied by $\left|V_{h i}\right|^{2}$. In the leading log approximation, we therefore substitute

$$
\log \left(m_{h}^{2}\right) \rightarrow \sum_{i}\left|V_{h i}\right|^{2} \log \left(m_{i}^{2}\right)
$$

Thus, the bound on the Higgs mass in the SM is replaced by a bound on a function of the masses and mixing angles. In particular, we can have heavy mass eigenstates up to $1 \mathrm{TeV}$ without violating the electroweak data.

In order to discuss the implications of our model on collider searches of the Higgs, we recall some issues regarding the search for the SM Higgs. Depending on the Higgs mass, there are several decay channels that are used to search for the Higgs. They are discussed at length in Ref. [1] and are summarized in figs. 22 and 23 there. Roughly speaking, we can say that

1. Through most of the mass range, the Higgs is searched for by looking into a resonance in different channels (like $H \rightarrow \gamma \gamma$ or $H \rightarrow Z Z^{(*)} \rightarrow 4 \ell$ ).

2. Around $m_{h} \gtrsim 170 \mathrm{GeV}$, where the dominant search channel is $H \rightarrow W W^{*} \rightarrow l \nu l \nu$, and again at higher mass $(\gtrsim 400 \mathrm{GeV})$, the Higgs is searched in a missing mass/momentum channels such as $H \rightarrow Z Z \rightarrow \ell \ell \nu \nu$ and $H \rightarrow W W \rightarrow \ell \nu j j$.

A relevant point to the Higgs search is the width of the Higgs, $\Gamma_{h}$. The experimental resolution is expected to be $\sigma \sim 2 \mathrm{GeV}$ [24] which is roughly the width of a Higgs with $m_{h} \sim 200 \mathrm{GeV}$. For $\Gamma_{h}<\sigma$ a reduction of the Higgs width due to added singlets is practically impossible to detected, while for $\Gamma_{h}>\sigma$ this effect is more noticeable.

Now we move back to our model. The main effect of our model on collider searches for the Higgs is that the cross section of each mass eigenstate is suppressed compared to a SM Higgs of the same mass. The leading production process at the LHC is gluon fusion through one-loop triangle diagram. Thus, the production cross section for each mass eigenstate is suppressed by a factor of $\left|V_{h i}\right|^{2}$. In the limit of many new singlets, $\left|V_{h i}\right|$ is small, and thus the cross section become very small.

The other effects depend on the parameters of the model. First consider the scenario where decays of the form $\phi_{i} \rightarrow 2 \phi_{j}$ are forbidden or negligible. Then, all the decay rates of the $i$ th mass eigenstate are suppressed by the same factor of $\left|V_{h i}\right|^{2}$. Thus, the branching ratios are the same as those of a SM Higgs with the same mass. The total width of each mass eigenstate is smaller by a factor of $\left|V_{h i}\right|^{2}$ compared to the width of a SM Higgs with the same mass.

In the case of a resonance search in the above scenario, the Higgs width affect our model. At low masses the Higgs width is small compared to the experimental resolution. Then the 
signal of each mass eigenstates is reduced by $\left|V_{h i}\right|^{2}$. (The width is also reduced by the same amount but this reduction cannot be noticed.) With many singlets, when $\left|V_{h i}\right|^{2}$ is very small for all $i$, the signal significance will drop below detection level.

At Higher masses, when the width of each mass eigenstate, $\Gamma_{i}$, is large, $\Gamma_{i}>\sigma$ the division of the signal between the singlets reduces the significance of each resonance by $\left|V_{h i}\right|$. The reason is that while the total signal is reduced by $\left|V_{h i}\right|^{2}$, this reduction simultaneously affects the width of the resonance.

In the case of a non-resonance search, the mass eigenstates contribute to the missing energy signal. Hence, the combined excess of these eigenstates over the background will be similar to that of a SM Higgs with $m_{h} \gtrsim 400 \mathrm{GeV}$. In this case it is possible to hide the Higgs signal by adding light mass eigenstates in the resonance search mass range.

Last we discuss the scenario where decays like $\phi_{i} \rightarrow 2 \phi_{j}$ are important. In particular, the interesting case is when all the heavy scalars decay almost entirely to the lightest one. In that case the situation is similar to the SM Higgs. Only one mass eigenstate is produced and its branching ratios are the same as a SM Higgs with the same mass. Yet, the production cross section and width are smaller than for a SM Higgs. This is because the production cross section for a heavy mass eigenstate is always less than half that of the light one. Thus, the fact that a heavy mass eigenstate decays into two light scalars cannot compensate for the reduction in the production rate and the parameter space allow for the possibility of the Higgs being hidden.

\section{EXAMPLES}

In the following we work out a few examples showing how additional singlets can hide the Higgs signal at the LHC. These examples are all within the suppressed $\phi_{i} \rightarrow 2 \phi_{j}$ scenario. In the first example we deal with a failure of a specific decay channel namely the $H \rightarrow Z Z \rightarrow 4 \ell$. The second example discusses the case of a missing mass channels such as the $W W^{*} \rightarrow \ell \nu \ell \nu$. In both examples we assume that the additional singlet masses are all in the range best suited for discovery in the discussed channel. Finally we give a third example which is the minimal solution for the LHC. In this example we follow the title of our paper by adding the minimal number of singlets needed to hide the Higgs at the LHC regardless of the search channel.

\section{A. $H \rightarrow Z Z \rightarrow 4 \ell$}

A search for the Higgs in this channel is most effective in the mass range $180-700 \mathrm{GeV}$. It is often dubbed the "golden channel" due to the expected high signal significance. If the Higgs mass is about $300 \mathrm{GeV}$ it is expected to yield a very high signal significance already in the first year of operation with as little as $10 \mathrm{fb}^{-1}$ of integrated luminosity. Failure to discover the Higgs with three times that luminosity seems unimaginable assuming a SM Higgs. However, 


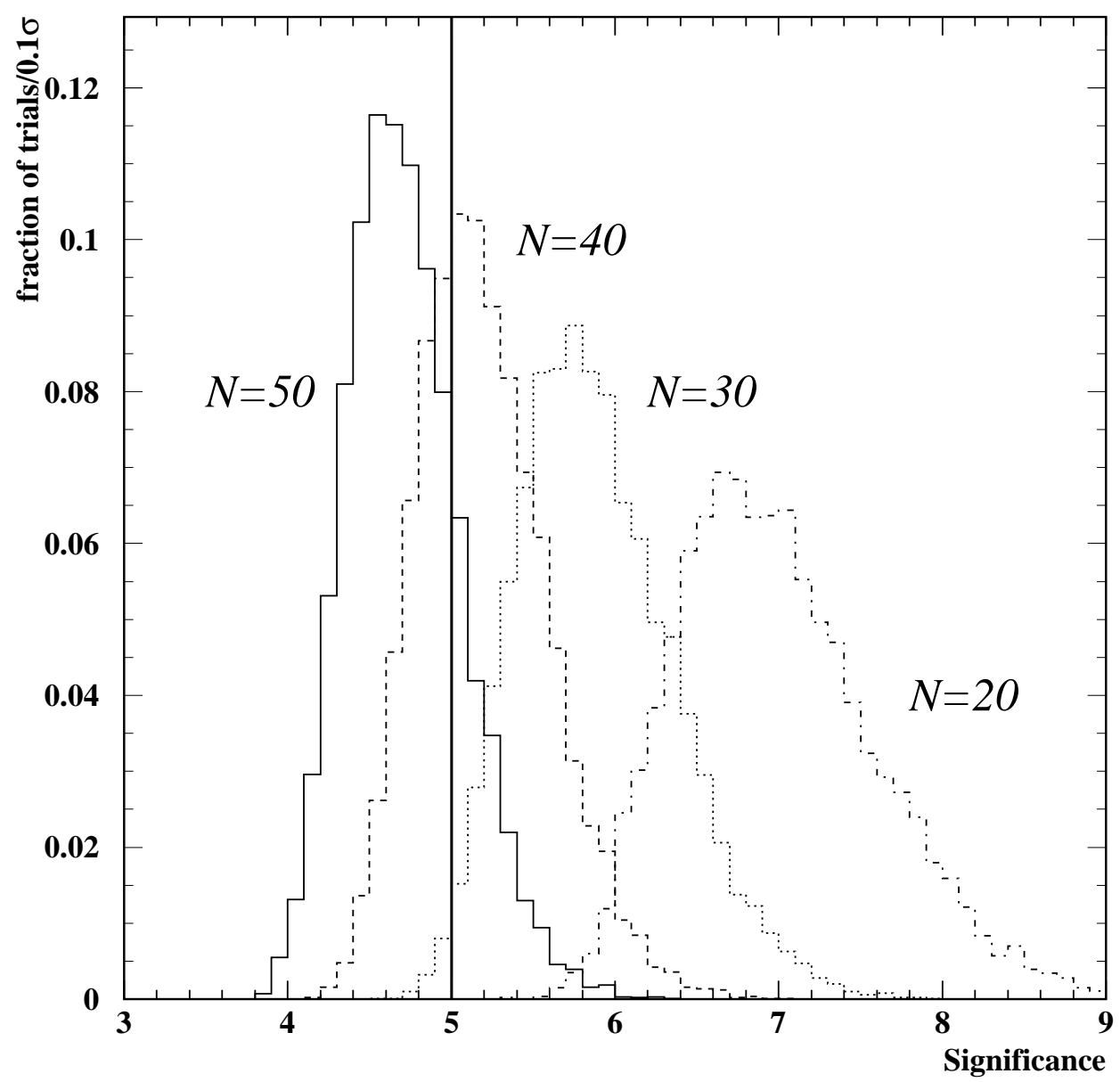

FIG. 1: Significance of the most significant singlet in 20-50 singlets toy Monte Carlo experiments. The vertical line at $\sigma=5$ represents the discovery threshold. See text for details.

if one adds 12 singlets in the mass region $200-300 \mathrm{GeV}$ with mixing constants $\left|V_{\text {hi }}\right|^{2}=0.03$ for $i=1 . .12$, additional 10 in the next $150 \mathrm{GeV}$ with $\left|V_{h i}\right|^{2}=0.04$ for $i=13 . .22$, and finally one more at around $600 \mathrm{GeV}$ with $\left|V_{h 23}\right|^{2}=0.24$ the signal significance drops below four at any given mass. This potential distribution of singlet masses was chosen with the Higgs width and experimental resolution in mind. However, while the mass spectrum will differ significantly from the expected SM Higgs signal it will also differ from the expected background shape and yield, indicating some "other" source. Figure1 shows the significance distribution of the most significant singlet in a $50(40,30,20)$ singlets experiment randomly drawn. The significance is calculated for a $30 \mathrm{fb}^{-1}$ of integrated luminosity for signal and background of the decay $H \rightarrow Z Z \rightarrow 4 l$ in the mass range $180-420 \mathrm{GeV}$. It can be seen that already with 30 arbitrary drawn singlets in that mass range, part of the simulated experiments have no singlet with more than $5 \sigma$ significance. The mixing constants, $\left|V_{h i}\right|^{2}$, 
were drawn from a Gaussian distribution whose mean was set to $1 /$ (number of singlets) and a width of half that number. If one were to include the higher mass range for this channel (420$700 \mathrm{GeV}$ ) and allow for a few singlets to occupy that region where the expected significance is lower, a larger fraction of the shown distributions will be found below $5 \sigma$ which is the case given in the above example. This is since the lower significance allow for larger value of $\left|V_{h i}\right|^{2}$ for the singlets in the high mass range.

\section{B. $\quad H \rightarrow W W^{*} \rightarrow l \nu l \nu$}

If the mass of all the singlets is in the vicinity of $170 \mathrm{GeV}$ where the dominant decay channel is $H \rightarrow W W^{*} \rightarrow l \nu l \nu$ the resulting signal will differ only slightly from the expected SM Higgs signal. This is due to the inability to fully reconstruct the Higgs mass. Hence the signal will be observed as an excess of event over the expected background. No possibility to hide the Higgs in this region if one insists on a solution of singlets solely in this mass range.

\section{Hiding the Higgs at the LHC}

Regardless of the specific examples above, the minimal solution in our model for hiding the Higgs for $100 \mathrm{fb}^{-1}$ will be with three singlets at about 118, 124 and $130 \mathrm{GeV}$ and about equal value for the three $\left|V_{h i}\right|^{2}$. In which case none of the mass eigenstates will be discovered and the overall number of observed events will be consistent with the background hypothesis.

\section{DISCUSSION AND CONCLUSIONS}

The Higgs boson is expected to be discovered at the LHC. Depending on its mass, different channels will be used to discover it. The standard model will be in a very bad position if the Higgs is not found. In this work we have shown that additional singlets might explain an absence of a Higgs signal without any signal of new physics. We analyzed scenarios corresponding to different masses in the range of $10^{2} \lesssim m_{i} \lesssim 10^{3} \mathrm{GeV}$. We assumed that all dimension-full parameters are of the order of the weak scale and all dimensionless parameters are of order one. In particular we asked how many singlets are needed in order to "hide" the Higgs. The answer depends crucially on the model parameters. In some cases, in particular when the mass eigenstates are close to $100 \mathrm{GeV}$, we found that as little as 2-3 singlets could reduce the significance below discovery level. In other cases, mainly when many of the masses are roughly above $300 \mathrm{GeV}$ we found that tens of singlets are needed to hide the Higgs.

We have concentrated on the Higgs search at the LHC. In fact, it could affect the searches for the Higgs also at LEP and the Tevatron and it is possible that the Higgs signal is hidden 
by a many-singlet solution. Yet, we did not investigate this issue in details. For the case of one extra singlet such a study was done in [15].

To conclude, we present a model in which the standard model Higgs field generates electroweak symmetry breaking but still the Higgs particle cannot be discovered at the LHC. Our model is very simple, and while it is not based on a well motivated theoretical framework, it serves as an example that the SM Higgs mechanism can escape detection at the LHC.

\section{Acknowledgments}

We are grateful to Ehud Duchovni, Andrey Katz, Amos Ori, and Witold Skiba for useful discussions and Yossi Nir, Yael Shadmi and Ze'ev Surujon for comments on the manuscript. The work of Y.G. is supported in part by the Israel Science Foundation under Grant No. 378/05. The work of Y.R. is supported in part by the Israel Science Foundation under Grant No. $1446 / 05$.

[1] L. Reina, "TASI 2004 lecture notes: Higgs boson physics," arXiv:hep-ph/0512377.

[2] R. Barate et al. [LEP Working Group for Higgs boson searches], Phys. Lett. B 565, 61 (2003) arXiv:hep-ex/0306033.

[3] LEP Collaborations et. al., Phys. Rept. 427, 257 (2006) arXiv:hep-ex/0509008.

[4] S. Asai et al. (ATLAS collaboration), Eur. Phys. J. C 32S2, 19 (2004) arXiv:hep-ph/0402254.

[5] J. Gunion, H. Haber, G. Kane, and S. Dawson, The Higgs Hunter's Guide, Addison-Wesley, Reading (USA), 1990.

[6] J. R. Espinosa and J. F. Gunion, Phys. Rev. Lett. 82, 1084 (1999) arXiv:hep-ph/9807275.

[7] J. J. van der Bij, Phys. Lett. B 636, 56 (2006) arXiv:hep-ph/0603082.

[8] D. G. Cerdeno, A. Dedes and T. E. J. Underwood, JHEP 0609, 067 (2006) arXiv:hep-ph/0607157.

[9] B. Patt and F. Wilczek, arXiv:hep-ph/0605188.

[10] X. P. Calmet, Eur. Phys. J. C 32, 121 (2003) arXiv:hep-ph/0302056.

[11] C. P. Burgess, M. Pospelov and T. ter Veldhuis, Nucl. Phys. B 619, 709 (2001) arXiv:hep-ph/0011335.

[12] J. J. van der Bij and S. Dilcher, Phys. Lett. B 638, 234 (2006) arXiv:hep-ph/0605008.

[13] R. Dermisek and J. F. Gunion, Phys. Rev. Lett. 95, 041801 (2005) arXiv:hep-ph/0502105.

[14] R. Dermisek and J. F. Gunion, Phys. Rev. D 73, 111701 (2006) arXiv:hep-ph/0510322.

[15] D. O'Connell, M. J. Ramsey-Musolf and M. B. Wise, arXiv:hep-ph/0611014.

[16] A. V. Manohar and M. B. Wise, Phys. Lett. B 636, 107 (2006) arXiv:hep-ph/0601212.

[17] A. V. Manohar and M. B. Wise, Phys. Rev. D 74, 035009 (2006) arXiv:hep-ph/0606172. 
[18] S. Chang, P. J. Fox and N. Weiner, JHEP 0608, 068 (2006) arXiv:hep-ph/0511250.

[19] V. Barger, P. Langacker, H. S. Lee and G. Shaughnessy, Phys. Rev. D 73, 115010 (2006) arXiv:hep-ph/0603247.

[20] T. Han, P. Langacker and B. McElrath, Phys. Rev. D 70, 115006 (2004) arXiv:hep-ph/0405244.

[21] V. Barger, P. Langacker and G. Shaughnessy, arXiv:hep-ph/0611112.

[22] M. Drees, Phys. Rev. D71, 115006 (2005) arXiv:hep-ph/0502075].

[23] M. E. Peskin and T. Takeuchi, Phys. Rev. D 46, 381 (1992).

[24] ATLAS collaboration, ATLAS Detector and Physics performance Technical Design Report, LHCC/99-14/15. 